\title{
DISTRIBUTIONS D'ÉNERGIE ÉLECTRIQUE
}

\section{I propos de la Durée des Autorisations de Voirie}

Note de ta Rédactron. - Nous donnons, sous ce titre, les pieces 'dont la lecture fera comprendre d'une façon plus complète les phases différentes par lesquelles l'esprit du législateur paraît avoir passé avant d'arriver au texte définitit dont nous avons donné le commentaire.

En premier lieu, on trouvera les travaux préparatoires de la loi, sous la forme du rapport fait à la Chambre des députés par M. Léon Janet.

Ce rapport donne, au dessous de chaque article, un exposé sommaire des motifs qui l'ont inspiré.

Ilais, comme il s'est plusieurs fois référé au rapport fait, pendant une précédente législature, par M. Guillain, il nous a paru absolument nécessaire de donner aussi ce second rapport qui, beaucoup plus complet quc le premier, a une importance de doctrine considérable.

Enfin, cette loi n'ayant eu qu'une seule fois l'honneur de la discussion en public - dans la suite elle a été seulement lue et adoptée - il nous a semblé bon de faire connaitre les observations, assez paures d'ailleurs, qu'elle avait fait naitre a la tribune.

On trouvera donc ci-joint les trois pièces annexes dont l'énumération a été donnée. (Voir l'article de notre collaborateur M. Paul Bovgault, no d'Avril rgir).

\section{CHAMBRE DES DÉPUTÉS}

IUUTHAM: LÉGISLATURE

Session de 1905

Antiexe au procès-verbal de la 2 séance du 13 juillel 1905.

\section{RAPFORT}

Fail au nom de la Commission des Usines Iydranliques ( $\mathrm{I}$ ) chargée d'examiner la proposition de M. Léon JANET sur les

\section{DISTRIBUTIONS D'ÉNERGIE}

\section{par M. Léon JANET, député}

Messicurs,

L.es applications de l'électricité ont complètement transfriné, depuis quelques années, l'activité industrielle de notre pays, el amené l'installation de grandes usines centrales à vapeur ou à gaz et d'usines hydrauliques.

L'ntilsation de celte forme de l'énergie, comprend, d'une parl, ha production de la force motrice et, d'autre parl, si: distribution sur les points oì elle doit être employéc.

(I) Celte Commission est composéc de MM. Paul Delombre prisident ; Guilain, Léon Janel, Ionoré Leygue (IIaute-Garonne), vice-présidents ; Buyat, François Carnot, Lebrun, Réville, secrélares ; Bouclot, Loque, Denys Cochin (Scine), le marquis de Laurcns-Castelet, Fernand David (Haute-Savoic), Sénac, Aynard, Cazeneuve, Vigouroux, Abel-Bernard, Deléglise, d'Iriart d'Echepare. - (Voir le $n^{\circ}$ 2III.)
La première question, tout au moins pour ce qui concerne la production de l'énergie dans les usines hydrauliques, a été l'objet de nombreux travaux parlementaires. En nous bornant à la législature acluelle, nous citerons : Ja proposition de loi présentée, le 3o mai 1 go3, par MM. Millerand et Pierre Baudin, et qui n'élail que la reproduction d'un projet de loi déposé, dans la précédente législature, par MM. Pierre Baudin, Ministre des Travaux publics, et Jean Dupuy, Ministre de l'Agriculture; la proposition de loi présentée le 23 juin igo3, par M. Guillain ; le rapport de M. Bouctot, au nom de la Commission d'initiative parlementaire, déposé lo 2o octobre ${ }_{9}$ o3 et concluant à la prise en considération de ces diverses propositions de loi, el à lcur renvoi à une Commission spéciale; le projel de loi de M. Mougcol, Ministre de l'Agriculture, relatif aux usines hydrauliques sur les cours d'eau non navigables ni llotables. Ce projet el ces proposilions de loi ont été renvoyces à une Commission de $22 \mathrm{mem}-$ bres, dite des usines hydrauliques.

L.es études de celte Commission ont montré que, s'il était utile d'arriver à réglementer la production de l'énergie électrique par les chutes d'eau, il était toul aussi indispensable de faciliter sa distribution, aussi bien dans le cas où elle a été produite par des chutes d'ean, que dans le cas où elle a élć produite par des moleur's à vapeur ou à gaz.

Cette question de la distribution de l'énergie, abstraction faite du mode de sa production, avait déjà donné licu à des travaux approfondis dans les législatures précédentes.

l.e 20 avril 189 亿, le Ministre des Travanx publies prit un arrêté pour instituer une Commission spéciale présidée par M. Armand housseau, consciller d'Btat, et clargé d'étudier. les dispositions d'un projet de toi relalif aux distributions d'énergie.

Cette Commission adressa son rapport au Ministre le 22 mai 1894 .

Lo projet de la Commission fut enyoyé aut Conseil d'Etat qui l'adopta le 22 février 1896 .

Le projet de loi fut déposé sur le bureau de la Chambre le 12 juillet 8897 . II donna lien à un rapport de M. Guillain, qui ful déposé le 8 fóvrier 1898 , mais ne put venir en discussion avant la fin de la législature $1893-1898$.

Dans ta législature $389^{8-1}$ go un nouvcau projet de loi a fart l'objet d'un rapport de M. Berthelol, déposé le 26 juin

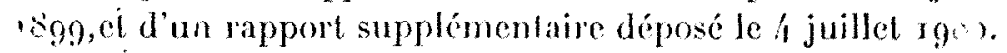
I.c projet de loi col venu en discussion devant la Chambie le ro juillet rgoo, mais il a été renvoyé à la Commission, et n'a pas été disculé à nouvcau avant la fin de la législature.

On comprend cependant le grand intérêt que présente celte question. Si tant de forces hydrauliques restent encore stériles, ce n'est pas seulement à cause des difficultés de leur utilisation sur place, mais ausi à cause des difficultés du 
transport de l'énergic produite. Les chutes d'cau se trouvent, le plus souvent, dans des régions montagneuses, où le transport des matières premières et des produits est très coûteux ; elles prendraient de suite une valeur beaucoup plus grande, si l'énergic produite pouvait facilement être transportée à des usines établies dans la plaine. I'utilisation économique de la houille ou du gaz de hauts fourneaux sur les lieux de production ne présente pas une moins grande importance.

Dans l'étal actuel de la législation, le transport de l'énergie ne peut s'opérer qu'en suivant les voies publiques, pour l'occupation desquelles des permissions de voirie, toujours précaires el révocables, peuvenl être accordées ou en s'entendanl à l'amiable avec les proprićtaires des terrains au-dessus desquels des conducteurs sont établis.

On comprend facilement toutes les difficultés que présente, dans de pareilles conditions, la création d'une industrie nécessitant d'imporlants capitaux de premier établissement.

L'auteur de la proposition de loi, qui en est en même temps le rapporteur, avait pensé qu'll y avait intérêt à chercher à remédicr à cettc situation, et notamment à donner, moyennant certaines charges, le bénéfice de l'utilité publique aux transports d'énergie présentant un véritable caractère d'intérêt général.

Ne tenant nullement à faire prévaloir ses idées personnelles, mais désirant simplement saisir de la question votre Commission des usines hydrauliques, il a repris le projet de loi de la précédente législature, annexé au rapport de M. Berthelot et modifié par un rapport supplémentaire, afin qu'il pût servir de base de discussion.

Votre Commission a reconnu d'abord que la réglementalion des distribulions d'énergic présentait un grand intérêt.

Elle a entendu les représentants les plus qualifiés des industries électriques en France et recueilli leurs observations au sujet du texte proposé.

Elle s'est immédiatement mise en rapport avec les Minislères intéressés, c'est-à-dirc les Ministères de l'Intérieur, du Commerce, des Travaux publics, des Finances et de l'Agriculture ; après de nombreuses et laborieuses réunions elle a adopté un texte qui a été accepté par les cinq Ministères précités.

Les projets de loi et les rapports déjà présentés sur ceite question dispensent votre rapporteur de refaire l'historique du tı ansport de l'énergic, et spécialement de l'énergie électrique. Toutefois, le projet de loi annexé au rapport de M. Berthclot ayant élé profondément modifié, il est néces saire d'indiquer les idées générales qui ont présidé à l'élaboration du nouveau texte que votre commission a l'honneur de vous soumettre daccord avec le gouvernement.

\section{Des divers transports d'énergie à soumeltre à la réglementation}

Le projet de loi annexé au rapport de M. Berthelot écartait de son domaine ct laissait simplement soumis aux règles de police, d'une part les transports d'énergie installés au moyen d'ouvrages fixes par un particulicr pour son usage personnel, d'antre part ies distributions d'énergie au moyen d'appareils transportés sur des véhicules : accumulateurs d'électricité, réservoirs d'air comprimé, etc. Par contre, il s'appliquait aux divers modes de transport de l'énergie par des ouvrages fixes, c'est-à-dire non seulement par des conducteurs d'électricité, mais encore par des conduites d'eúu à haute pression ou d'air comprimé. On peut se demander à défaut d'indication précise, si la Commission de la précédente législature avait cntendu la rendre applicable aux distributions de gaz.

Il a paru à votre Commission qu'il était préférable de ne viser dans la nouvelle loi que les distributions d'éncrgie électrique, à cause de la difficulté de trouver pour certains articles une rédaction applicable aux distributions de toute nature.

Par contre, il a paru nécessaire de soumettre à la nouvelle législation, non seulement les entreprises ayant pour objet la distribution de l'énergie électrique au public, mais encore celles n'intéressant que les particuliers.

Le texte annexé au rapport Berthelot laissait subsister les dispositions de la loi du 25 juin 189 , concernant l'établissement des conducteurs d'energie électrique qui n'étaient pas contraires à celles de la nouvelle loi. Il a paru préférablo à votre Commission d'inscrire dans son texte les articles de la loi du 25 juin 1895 qui étaient conservés, de manière à pouvoir abroger en bloc la loi en question. Il a été difficile de faire accepter cette solution par l'Administration des pos. tes et télégraphes, qui désirait ne pas remettre en discus. sion le paragraphe $1^{\text {er }}$ de l'article 2 de cette loi, spécifiant que les conducteurs aériens ne pouvaient être établis dans une zone de dix mètres en projection horizontale de chaque côté d'une ligne télégraphique ou télëphonique sans entente préalable avec elle, et l'adhésion qu'elle a donnée finalement à l'ensemble de la proposition de loi a été expressément subordonnée au maintien dé la disposition en question.

La solution proposée par votre Commission aura l'avantage de codifier en un seul texte toutes les dispositions légales concernant les distributions d'énergie électriques.

\section{Des divers régimes sous lesquels peuvent être plackes les distributions d'énergie}

Votre Commission s'est efforcée de maintenir à l'industrie électrique toutes les libertés dont elle dispose actuellement. Elle a cherché, en outre, à faciliter, pour l'avenir, l'établissement des distributions d'énergie, en leur donnant une stabilité dont elles ne jouissaient pas jusqu'à préscnt.

La première catégorie des transports d'énergie électrique est celle qui bénéficie des dispositions de l'article premier de la loi du 25 juin 1895 ; les conducteurs électriques qui ne sont pas destinés à la transmission des signaux et de la parole peuvent être établis, en dehors des voies publiques, sans autorisalion ni déclaration, à la condition toutefols de ne pas s'approcher à moins de ro mètres des lignes télígraphiques ou téléphoniques préexistantes.

Une deuxième catégorie comprend les conducteurs d'énergis électrique qui ne peuvent être établis, sur des terrains privés, qu'avec une autorisation; ce sont ceux qui sont visés par l'article 2 de la loi du 25 juin 1895 , comme devant s'approcher à moins de dix mètres de distance horizontale d'une ligne télégraphique ou téléphonique. Votre Commission a pensé que c'était le Préfet qui devait continuer, comme aujourd'hui, à délivrer ces autorisations. 
Les deux calégories ne figuraient pas dans le projet de loi annexé au rapport de $M$. Berthelot, parce qu'il ne visait que les distributions de l'énergie au public, et que les ouvra. ges fixes de ces contributions devaient nécessairement empruntcr les voies publiques servant daccès aux propriétés prives à desservir

Une troisième catégorie comprend les distributions d'éner. gie qui empruntent des voies publiques en vertu de permis. sion de voiric. On sait que toute occupation de la voie pu blique par des ouvrages fixes établis au-dessus ou au-dessou: lu sol, esl surbordonnéc à une permission de voirie qui es donnce par le Préfet pour les routes nationales et départe menlales, les chemins de grande communication et d'inté rêt commun, par le maire pour les voies urbaines et pous les chemins vicinaux ordinaires et ruraux. La permission de voirie, précairc et révocable, ne peut régler qué les con. ditions d'occupation, dans les rapports avec la voirie.

C'est ce régime des permissions de voirie qui a permi: a un grand nombre de transports de force de s'établir su le territoire français. Les industriels tiennent essentiellement à ce qu'il soit conservé dans la législation à intervenir.

La question doit évidemment ètre tranchée dans un sens favorable pour les distributions d'énergie qui ne s'adressent pas au public.

Elle est plus discutable pour les distributions publiques d'ínergie. Le projet de loi déposé par le Gouvernement en ${ }_{1} 898$ donnait le droil à l'Administration d'imposer à toul entrcpreneur de distribution publique d'énergie, empruntant les voies publiques, le régime de la concession, c'està-dire des obligations précises erivers les habitants, soit pour le prix de vente de l'énergie, soit pour les autres conditions de la fourniture.

La Commission de la précédente législature, tout en approuvant ces règles en principe, avait pensé que les termes trop absolus du projet du Gouvernement pouvaient empếcher bien des distributions utiles. Selon elle, les entreprises ayant pour objet de distribuer l'énergie au public devaient en règle générale, être soumise aux obligations d'un cahies des charges, mais elle admettait que l'on fit des exceptions.

Votre Commission a pensé que, même pour les distribu. lions publique d'énergie, il y avait lieu de laisser les industriels opter entre le régime des permissions de voirie et celui des concessions.

La quatrième catégorie de distributions d'énergie électri que comprend celle des concessions simples sans déclaratior. d'utilité publique.

Les conditions dans lesquelles sont données ces conces sions restent à peu près les mêmes que dans le projet de lo: de la précédente législature. Toutefois, il a paru à votre Commission qu'il fallait étendre aux syndicats de communes le droit de concéder les distributions d'énergie électriques établies sur le territoire des communes syndiqués. La question gavait été soulevée par M. Vaillant dans la séance du to juillet r goo lorsque le projet de loi était venu en discussion devant la Chambre, et la précédente Commission s'élait montrée disposée à donner satisfaction à ce desideratum.

la cinquième catégorie de distributions d'énergie comprend celle des concessions déclarées d'utilité publique par un décret en Conseil d'Etat.
Sous le régime actuel, il n'est pas possible de donner aux ouvrages des entreprises de transport el de distribution de l'énergie électrique le caractère de travaux publics et lo bénéfice de la déclaration d'ulilito publique, si ce n'est par des lois d'espèce, comme celle du 9 juillet $18_{92}$, relative à une distribution d'ćnergie électrique produite par une chute d'eau dérivée du Rhône en amont de Lyon ; d'ailleurs, en l'absence d'une loi organique sur la matière, les demandeurs se trouvent exposés à voir l'instruction de leurs projets indéfiniment relardée par les hésitations des diverses administrations qui ne savent comment résoudre les questions si complexes que ces projets soulèvent.

La déclaration d'utilité publique donnera le droit d'appui et de passage sur les propriélés privécs ; en éehange, ello pourra imposer certaines obligations, notamment le relour des ouvrages à l'autorité concédante à la fin de la concession, qui sera toujours temporaire, et établir certaines obligations envers le public, quant aux prix de la fourniture d'énergie, et quant aux autres conditions de cette fourniture.

En ce qui concerne les deux dernières catégories de distributions d'éncrgie, une discussion très approfondie s'est élevée au sein de la Commission pour définir les principales obligations susceptibles d'ètre imposécs par l'acte de concession.

Les industriels qui ont été entendus ont vivement insisté pour que la plus grande liberté soit laissée à la venle du couront. Ils ont demandé à ne pas être forcés de soumettre les tarifs à une homologation quelconquc.et onl même manifesté quelques rejpugnances au sujet de la fixalion d'un prix maximum pour la fourniture de l'énergie, prétendant que ce maximum n'était ulile que si la législation permetlait, au profit d'un concessiomnaire délerminé, l'établisscment d'un monopole.

Le texte annexé de $M$. Berthelot parlait à l'article 2 de "concession avec cahier des charges et larif maximum" et laissait, en vertu de l'article r $r$, à un règlement d'administration publique le soin de déterminer "les formes de l'homologation des tarifs par' l'autorité concédante".

Votre Commission a reconnu qu'il serait regrettable de donner le même régime aux tarifs d'énergic élcetrique qu'aux tarifs de chemin de fer, en ctablissant l'homologation comme règle.

Les tarifs que fixent Ins Sociétés actuclles de distribution d'énergie dépendent d'un grand nombre d'éléments, parmi lesquels les principaux sont : l'heure de la demande, la quantité demandée, et la régularilé. Il scrait à peu près impraticable d'arriver à une tarifications rationnclle tenant comple de ces divers éléments.

On peut craindre sans doute qu'avec la liberlé des tarifs, une entreprise industrielle n'obtienne pas le courant qui lui est nécessaire à aussi bon compte qu'une autre entreprisc placée dans des conditions analogues. C'est là une objection dont l'imporlance n'a pas échappé à votre Commission. Celle-ci a pensé cependant qu'clle n'était pas de nature à exiger l'application du principe de l'homologation, attendu que l'énergie électrique peut, en cas de besoin, être produite finalement par tout industriel, pour son usage persornel, al moyen d'un moteur à vapeur. 
D'un autre còlé, votre Commission a cstimé qu'il n'étail pas possible d'aller jusqu’à la liberté absolue rédaméc par divers industriels, el qu'un larif maximum aumat l'avanlage de prérenir rertains abus. sans apporter d'entraves séricueco au développenent de l'industrie.

Une autre question qui a molivé une discussion approfondic at sein de volle Commission est celle du monopole. Convenait-it que l'acto de concession pùt permetle ou interdire, soit d'une nanière absolue, soit pendant une période détcrminée, l'aulorisation d'inslallations concurrentes ?

Le texte annexée au rapport de M. Berthelof portail qu'aucunc concession ne pouvail fairc obstacle à ce qu'il soit accordé des permission ou concessions concurrentes, que loulefois un monopole pouvait être institué pendant une durée lımiléc à quinze ans à partir de l'expiration du délai fixé pour le commencement de la mise en exploitation.

Le but de cette rédaction était d'empêcher certaines communes de se lier avec une société concessionnairc pour une trop longue durée, ainsi que cela est arrivé souvent avec des sociélés d'éclairage par le gaz. Il devient alors impossible à la commme de profiter des progrès qui sont réalisés, avant l'expiration de la concession. C'est là une des circonstances qui a retardé le plus linstallation de l'éclairage élcctrique dans un grand nombre de villes.

Les industriels qui ont été entendus se sont divisés sur celte question; ils se sont montrés généralement peu favorables à un monopole trop prolongé ; selor eux, l'industrie électrique a cependant besoin d'être assurée que, pendant un cerlain nombre d'annćes, il ne sera pas possible à une municipalité mal disposéc pour clle de la ruiner en accordant à une société concurrente un traité plus avantageux.

Votre Commission a examiné successivement les diverses solutions susceptibles d'être adoplées, monopole plus ou moins prolongé pour toules les calćgories d'énergie, ou pour l'ćclairage seul, à l'exclusion de la force motrice.

Elle a reconnu que linterdiction ou la limitation trop étroite du monopole empècherail beaucoup de petites communcs de trouver un concessionnaire pour la fourniture de l'énergie destinée à l'éclairage public et privé, ou du moins les obligerail ì payer trop cher l'éncrgie produite. Il est souvent de toule impossibilité que, dans ces petites communes, deux entreprises similaires puissent vivre. Dans diverses régions, beaucoup de communes ont accordé des concessions pour la foumiture de l'énergie électrique pendant une durée de trenle ans, avec monopole de fait pendant toule celte période.

Votre Commission a donc pensé qu'il y avait lieu de faire une distinction entre l'énergie destinée à la force motrice, qui ne pourrait en aucuu cas faire l'objet d'un monopole, et l'énergic destinée à l'éclairage qui pourrait, au contraire, constituer un monopole pendant toule la duréc de la concession. La limite de $\mathbf{1 5}$ ans élait évidemment insuffisante ; votre Commission l'avait d'abord portée à 25 ans puis à 30 ans, el enfin elle a fait disparaître tout délai pensant qu'aujourd'hui l'attention des communes avait été suffisamment appelée sur les inconvénients que présentaicnt de trop longues concessions avec monopoles.

Organisation du contrôle des distributions d'énergie

Le texte annexé au rapport de $M$. Berthelot laissait, par son article 2 , ̀̀ un règlement d'administration publique le soin de détermincr l'organisation du conlròle dont les frais Jevaicut être à la charge du concessionnaice.

Celte organisation élait une des questions présentant lo hus de difficultés, en raison des interventions nócessaires te l'Administration des postes el télégraphes pour la procetion des fils télégraphiques el téléphoniques, des services e voiric pour la surveillance des fils ćtablis sur les voies pu,liques.

Il a paru à volre Commission que c'était au législuleur [u'il appartenail de trancher, au point de vue des principes, celte question d'organisation du contrôle, en n'abondonnant au règlement d'administration publique que les délails de celte organisation.

Après avoir entendu les représentants des Ministres des Travaux publics, de l'Intérieur et du Commerce, votre Commission a reconnu qu'il serait pratiquement impossible de soumettre les industriels à deux ou trois contrôles différents, dont chacun pourrait leur donner des indicalions contradictríres, et que J'unité de contrôle s'imposail.

Il lui a paru, d'autre part, que, étant donnée la nature du contrôle à exercer, il serait logique de le placer sous l'autorité du Ministre des Travaux publics.

L'Administration des postes et télégraphes gardera naturellement la haule main sur les questions de protection des fils télégraphiques et téléphoniques, mais elle devra faire comme une autre administration publique qui aurait à so plaindre du voisinage d'une ligne de distribution d'énergie, el adresser ses observations, non pas directement au concessionnaire, mais au service du contròle constitué comme il vient d'être dit

Ces dispositions ont été acceptées par les Ministres intéressés.

\section{Examen des articles de la proposition de loi}

Nous avons indiqué plus haut Ies principes des modificalions essentielles qui avaient élé apportées par votre Commission au texte de la précédente législature.

De nombreuses modificalions de détail ont été en oulre introduites dans la nouvelle proposition de loi. La raison d'être de ces changemenis scra donnéc à propos de l'cxameì des articles, que nous allons maintenant aborder.

\section{TITRE $1^{\text {sT }}$}

CI.ASSIFICATIOA DES DISTRIBUTIONS D ÉNERGIE ÉLECTRIQLE

Article premier. - Les distributions d'énergie électrique qui ne sont pas destinées à la transmission des signaux et de la parole et auxquelles le décrel-loi du 27 décembre 1851 n'est pas, dès lors, applicable, sont soumises pour leur tátblissement et leur Jonctionnement anx conditions générales ci-après :

L.es modificalions apportées à l'article premier du textc de la préccdente législature résultent :

I $^{\circ}$ De ce que la nouvelle proposition de loi ne s'applique plus, comme nous l'avons déjà dit, qu'aux distributions d'ćnergie électrique, au lieu de viser toutes les distributions. d'énergie ;

$2^{\circ}$ De ce.qu'elle s'applique, par contre, aux transports d'énergie qui ne donnent pas lieu à une distribution au public. 
Art. 2. - Une distribulion d'énergie électrique n'empruntant en aucun point de son parcours des voies publiques peut être établie cl exploitée, soit sans autorisation ni déclaration, soit lorsque ses conducteurs doivent ĉtre établis, en un point quelconque, à moins de dix mèlres de dislance horizonlale d'une ligne télégraphique ou téléphonique, préexislante, en verlu d'une autorisalion délivrée dans les conditions spécifiées au titre II de la présente loi.

Cotle rédaction est entièrement nouvelle ct vise une catégoric de transports d'énergie à laquelle ne s'applique pas lo lexte de la précédente législature, puisque, toutes les fois qu'on faisait une distribution au public, on était obligé d'mprunter des voies publiques.

Art. 3. - line distribution d'énergie électrique empruntant sur tout ou parlie de son parcours les voies publiques peul être exploilće, soit en vertu de penmissions de voirie, sans durée déterminée, dans les conditions spécifiées au titre III de la présente loi, soit en veriu de concessions d'une durée déterminée, avec cahier des charges et tarif maximum, dans les conditions spéciales au titre $I V$, s'il n'y a pas déclaration d'utililé publique, ou dans celles spécifiées au titre $V$, s'il y a déclaration d'ulilité publique.

Elle peut, suivant la demande de l'entrepreneur, ĉtre soumise simultanément dans des communes différentes à des régimes différents, soil celui des permissions de voirie sur une partie de son réseau, soit celui de la concession simple ou celui de la concession déclarée d'utililé publique dans d'aulres parties.

Colte rédaction est lrc̀s différente de celle de l'arlicle 2 du projel de la précédente législature, auquel il correspond.

Votre Commission a tenu à laisser bénéficier les cntreprises nouvelles de la faculté qu'clles possèdent aujourd'hui d'établir des distributions d'énergie au moyen de permissions de voirie.

Elle a même spécifié que l'unité de régime n'était pas obligaloire et q̀u'une entreprise pouvait être placée sous des régimes différents, pour diverses parties de son réseau.

\section{TITRE II}

DES OUVRAGES DE TRANSPOTT ET DE DISTRIBLTION D'ÉNERGIE B́LECTRIQUE ÉTABLIS EXCLUSIVEMENT SUR DES TERRAINS PRIVÉS SOUS LE RÉCIME DES AUTORISATIONS.

Art. 4. - Tes aulorisations prévues par l'article 2 sont délivrées par le préfet, en conformité de l'avis émis par l'administration des posles el télégraphes et dans un délai de liois mois à partir de la demande.

Les installalions visées dans ces autorisations devront salisfaire aux conditions techniques déterminées jar les arrètés prévus à l'article 19 de la présente loi.

Eiles devront être exploitées et entretcnues de manière à n'apporter par induction, dérivation ou aulrement, aucun trouble dans les transmissions télégraphiques ou téléphoniques par les lignes préexislantes.

Lorsque, pour prévenir ou faire cesser ce trouble, il sera nécessaire d'exiger le déplacement ou la modification des lignes préexistantes et en cas de non-enlente avec l'exploilant, la nature des travaux à exéculer sera déterminée par le ministre du commerce, de l'industrie, des postes et des télégraphes, après avis du comité d'électricité visé par l'ar- ticle 20. Dans tous les cas, les frais nécessilés par ces déplacemenls ou modifications seront à la charge de lexploilanl.

Cet article, entièrement nourcau, indique la procédure a lacuelle seront sounises les distribulions d'énergie dont il est parlé à l'arlicle 2, qui n'cmpruntenl, en aucun point de leurs parcours, des voies publiques, mais doivent être élablies à moins de dix mètres de distance horizontale l'une ligne télégraphique ou téléphonique précxistante.

Les dispositions de cet article s'inspirent de celles de la loi du 25 juin $x 895$, concernant l'élablissement des conducteurs d'énergic éleclrique autres que les conducleurs lólégraphiques et téléphoniques, ct qui est abrogćc par le nouveau texte.

Le delaj de trois mois indicué au premier paragraphe est colui prévu pa l'article 2 de la loi du 25 juin 1895 .

Le second paraglaphe renvoie, pour les condilions lechniques à remplir, aux articles visés par l'article sg du nouveau lexte.

Le troisième el le quatrième paragraphes ne fonl que reproduire, avec quelques modilications de délail, l'arlicle 7 de la loi du 25 juin 1895 .

\section{TITRE III}

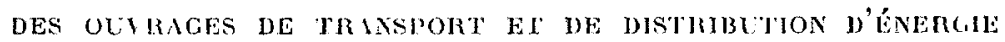
ELECTRQUE SOUS LE HEGME DES IEHMISSJONE DE VOMAE

Art. 5. - Les permissions de voirie sont délivrées par le préfet ou par le maire, suivañt que la voie empruntéc rentre dans les attributions de l'un ou de l'autre, sous les condilions ordinaires des arrêtés réglementaires relatifs à ces permis"sions, et en ontre sous les condilions stipnlées par les règlements d'administralion publique visés à l'arlicle 18 de la présente loi.

Elles ne peuvent prescrire aucune disposilion relalive aus conditions commerciales de l'exploilalion.

Elles ne penvent imposer an permissiomaire aucune charge pécuniaire aulre que les redevances prévues au paragraphe 7 de l'article 18.

Aucune permission de voirie ne peul faire obslacle à ce qu'il soit accordé sur les mèrres voies des permissions ou concessions concurrentes

Le but de cel arlicle, qui est nouveau, est d'indiquer qu'il n'est rien innové au régime actuel des permissions de voirie.

Yotre Commission a tenu à spécifier qu'il ne serait pas admissible de subordonner la permission de voirie à des conditions commerciales déterminces de vente du courant, on au payement de redevances autres que celles gui sont lues éfuitablenient en raison de l'occupation du sol des voics publiques.

Par contre, il ne scra pas permis de conférer un monopole quelconque au moyen de permissions de voiric.

\section{TITRE IV}

RÉGIME DES CONCESSIONS SIMPIES SANS DĹCHARATION D'UTILITÉ PUBLIQVE

Art. 6. - I. a concession d'une distribution publique l'énergie cst donnée, après enquête, soil par la commune ou ar le syndicat formé entre plusiears communes, si la demande de concession ne vise que le territoire de la commune ou du syndicat, soit par l'Etat dans les autres cas. 
Toule concession est solumise aux clauses d'un cahier aes charges conforme à l'un des lypes approuvés par décrel délibere en Conseil d'Elat, sauj les derogalions ou modificalions qui seraient expressément formulées dans les conventions passécs au sujet de ladile concession.

Cetle rédaction est tout à fait analogue à celle de l'article 3 du lcrute de la précédente législature, sauf en ce qui concerne l'extension aux syndicats de communes des pouvoirs concédés aux communes. Il a déjà été dit que, lors de la discussion en séance publique, cetle extension avait été acceptée en principe.

Art. 7. - Lorsque la concession esl de la compétence de l'Elat, l'acle de concession est passé par le préfet, si elle ne s'élend que sur des communes situées dans le teritoire da département, ou par le ministre des travaux publics, après avis du ministre de l'intérieur, si elle s'élend sur des communes situées dans plusieurs départements.

Lorsque la concession est de la compétence de la commune, l'acte de concession est passé par le maire, en exéculion d'une délibération du conseil municipal.

Si la concession est de la compétence d'un syndicat de communes, l'acte de concession est passé par le président du comité du syndicat, en exécution d'une délibération de ce comité, homologuée par des délibérations des conseils municipaux de toutes les communes syndiquées.

La concession donnée au nom de la commune ou du syndicat de communes n'est définitive qu'après avoir été approuvée par le préfet.

T'outejois, si l'acte de concession passé par le ministre, le préfet, le maire ou le président du comité du syndicat de communes comporte des dérogations ou modifications au cahier des charge type, il ne devient definitif qu'après avoir' été approuvé par un décret délibéré en Conseil d'Etat.

Cetle rédaction ne fait que reproduire l'article 4 du texte - de la précédente législature en tenant compte de la faculté donnée par l'arlicle précédent aux Syndicats de communes.

Pour les concessions de l'Etat, c'est une règle à peu près génćrale, dans l'élal actuel de notre décret administratif, qu'elles soient donnóes par une loi ou un décret délibéré en Conseil d'Etat. Il y a cependant quelques exceptions à cette règle. Ainsi, les décrels du 25 mars 1852 et du $\mathrm{I} 3$ avril $\mathrm{I} 86 \mathrm{r}$ sur la décentralisation administrative ont remis au Préfet le pouvoir de concéder l'établissement de débarcadères publics sur les bords des fleuves et rivières, et de fixer lcurs tarifs et conditions d'exploitation. Il a semblé à votre Cómmission, comme à celle de la précédente législature, qu'il y avait là un exemple à imiter, et qu'il convenait de laisser, suivant qu'un seul ou plusieurs départements sont intéressés, au Préfet ou au Ministre des Travaux publics la faculté d'accorder les concessions ayant un cahier des charges conforme à l'un des types généraux. On n'aura recours au Conseil d'Etat que lorsque l'acte de concession comportera des modifications ou des dérogations à l'un des cahiers des charges types.

Les industriels auraient désiré que cette formalité ne fût imposée qu'en cas de dérogations importantes. Il n'a pas paru possible à votre Commission d'insérer une disposition aussi peu précise dans un texte de loi. Toute dérogation, si minime qu'elle soit, nécessitera donc un décret en Conseil d'Etat.
Art. 8. - Aucune concession ne peut faire obstacle à ce qu'il soit accordé des permissions de voirie ou une concession à une entreprise concurente, sous la réserve que celle-ci n'aura pas des condilions plus avantageuses.

Toulefois, lacle par lequel une commune ou un syndical de communes donne la concession de l'éclairage public el privé sur tout ou partie de son territoire peut stipuler que le concessionnaire aura seul le droit d'uliliser les voies publiques dépendant de la commune ou des communes syndiquées dans les limites de sa concession, en vue de pourvoir à l'éclairage privé par une distribution publique d'énergie, sans que cependant ce privilège puisse s'étendre à l'emploi de l'énergie à tous usages autres que l'éclairage, ni à son emploi accessoire pour l'éclairage des locaux dans lesquels l'énergie est ainsi ulilisée.

Pendant la durée du privilège ainsi institué, les permis. sions de voirie délivrées par le préfet et les actes de concession passés au nom de l'Etal devront tenir compte de ce privilège dans les obligations imposées aux permissionnaires et concessionnaires.

Cette rédaclion présente de notables différences par rapport à celle de la première partie de la précédente législature, qui autorisait la constitution d'un monopole pendant les quinze premières années de la concession.

Ainsi que cela a déjà été dit au début de ce rapport, votre Commission a écarté d'une manière absolue la constitution d'un monopole pour la force motrice, l'électro-chimie, l'électro-métallurgie, etc., mais dans le but de faciliter l'éclairage des petites communes, elle a autorisé celles-ci à constituer un monopole d'éclairage pour toute la durée de la concession.

Elle a lenu d'ailleurs à spécifier que ce monopole ne saurait s'appliquer non plus à l'éclairage accessoire des locaux industriels. Toutefois, pour éviter les abus, pour empêcher, par exemple, qu'un commerçant consommant de l'énergie pour actionner un petit moteur, ne puisse faire passer pour accessoire l'éclairage d'un grand magasin, il a été entendu qu'il ne pouvait être question que de l'éclairage accessoire des locaux dans lesquels l'énergie est utilisée pour force motrice, électro-chimie. etc.

Art. 9. - L'acte de concession ne peut imposer au concessionnaire une charge pécuniaire autre que les redevances prévues au paragraphe 7 de l'article 18, ni attribuer à l'Etal ou d̀ la commune des avantages particuliers autres que les prix réduits d'abonnements qui seraient accordés aux services publics pour des fournitures équivalentes.

Cet article est la reproduction du dernier paragraphe de l'article 5 du texte de la précédente législature auquel on a seulement ajouté à la fin les mots " pour des fournitures équivalentes " de manière à mieux préciser le sens de la disposition.

Il a pour objel d'empêcher l'Etat et les communes de se consiluer abusivement des ressources budgétaires au détriment des concessionnaires et, par suite, au détriment des abonnós des distributions d'énergie.

Art. Io. - La concession confère à lentrepreneur le droult d'exécuter sur les voies publiques et leurs dépendances, tous travaux nécessaires à l'établissement et à l'entretien des ouvrages en se conformant aux conditions du cabier des charm 
ges, des règlements de voirie et des règlements d'administration publique prévus à l'article 18 ci-après.

L'autorité qui a fait la concession a toujours le droit, pour un motif d'intérêt public, d'exiger la suppression d'une partie quelconque des ouvrages d'une concession ou d'en faire modifier les dispositions et le tracé.

L'indemnité qui peut être due, dans ce cas, au concessionnaire, est fixée par les tribunaux compétents si les obligations ét droits de celui-ci ne sont pas réglés soit par le cahier des charges, soit par une convention postérieure.

Ces dispositions ne figuraient pas dans le texte de la précédente législature, mais elles se trouvaient dans le projet de loi élaboré par le Conseil d'Etat en xgo6. Elles précisent divers points qui ne sont pas sans importance, et notamment le droit, pour l'autorité qui a fait la concession, d'exiger, dans l'intérêt public, la suppression ou la modification de certains ouvrages déjà établis.

l.e texte du Conseil d'Etat faisait fixer l'indemnité pourant être due au concessionnaire par une commission spéciale de trois membres. Votre Commission a cru préférable de confier celte attribution aux tribunaux compétents.

\section{TITRE V}

RÉGIME DES CONCESSIONS DÉCLARÉES D'UTILTTÉ PUBLIQUE

Art. II. - Sont applicables aux concessions déclarées d'utilité publique l'article 6 , les paragraphes $1^{\text {er }}, 2$ et 3 de l'article 7 et les articles 8,9 et 10 de la présente loi.

La déclaration d'utilité publique est prononcée, après enquête, par un décret délibéré en Conseil d'Etat, sur le rapport des ministres des travaux publics et de l'intérieur, après avis du ministre du commerce, de l'industrie, des postes el des télégraphes et du ministre de l'agriculture.

L'acte de concession ne devient définilif qu'après avoir été approuvé par ce décret.

Lc premier paragraphe de cet article a pour but d'indiquer, alin d'éviter des répétitions, toutes les dispositions concernant les concessions simples, qui sont applicables aux concessions déclarées d'utilité publique.

Les paragraphes 2 et 3 indiquent la forme dans laquelle sera prononcée la déclaration d'utilité publique. Cette forme est d'ailleurs analogue à colle qu'établissait l'article 6 du texte da la prérédente législature.

Il ne s'agit ici, bien entendu, dans cet article, que des ourrages destinée au transport et à la distribution de l'énergie électrique.

Ainsi la déclaration d'utilité publique des ouvrages destinés à la production de l'énergie par des moteurs à vapeur ou à gaz, ne pourra, en aucun cas, être prononcée par un décret rendu en excicution de la présente loi.

Quant à l'établissement des ouvrages hydrauliques destinés à la production de l'énergie électrique, tels que barrages, canaux de dérivation et de fuite, usines hydrauliques, etc., ha déclaration d'utilité publiq̣ue est traitée dans le projet de loi spécial déposé au nom du Gouvernement par M. le Ministre de l'Agriculture au sujet des usines hydrauliques à établir sur les cours d'eau qui ne sont ni navigables ni flottables.

Pour que la question fût complètement résolue, il aurait ité utile que M. Ie Ministre des Travaux publics déposât un projet analogue à celui de M. le Ministre de l'Agriculture, au sujet des usines lyydrauliques à établir sur les cours d'eau navigables et flottables.

Il convient d'ailleurs de noter que l'établissement des usines hydrauliques a déjà étć envisagé d'une manière génćrale dans deux propositions de loi déposées au cours de cette législature, émanant, l'une de M. Guillain et l'autre de MM. Millerand et Baudin.

Art. 12. - La déclaration d'ulilité publique investit le concessionnaire, pour l'exécution des travaux dépendant de la concession, de tous les droits que les lois et règlements confèrent à l'Administration en matière de travaux publics. Le concessionnaire deméure en même temps soumis à toutes les obligations qui dérivent, pour l'administration, de ces lois et règlements.

S'il y a lieu à expropriation, il y est procédé conformément à la loi du 3 mai 1841, au nom de l'autorité concédante et aux frais du concessionnaire.

La déclaration d'ulilité publique d'une distribution d'énergie confère, en outre, au concessionnaire, le droit:

$1^{\circ}$ D'établir à demeure des supports el ancrages pour conducteurs aériens d'électricité, soit à l'extérieur des murs ou façades donnant sur la voie publique, soit sur les toits et terrasses des batiments, à la condition qu'on y puisse accéder par l'extéricur, élant spécifié que ce droit ne pourra êlre exercé que sur les conditions prescrites, tanl au point de vue de la sécurité qu'au point de vue de la commodité des habitants, par les règlements d'administralion publique pré. vus à l'article 18 , lesdits règlements devant limiter l'exercice de ce droit au cas de courants électriques tels que la présence desdits conducteurs d'électricité à proximité des bátiments ne soit pas de nature à présenter, nonobstant les précautions prises conformément aux règlements, des dangers graves pour les personnes ou les bâtiments ;

$2^{\circ}$ De faire passer les conducteurs d'électricité au-dessus des propriétés privées, sous les mêmes conditions et réserves que celles spécifiées à l'aliéna $1^{\circ}$ ci-dessus :

$3^{\circ} D^{\prime}$ établir à demeure des canalisalions soulerraines, ou des supports pour conducleurs aériens, sur des terrains privés non bâlis, qui ne sont pas fermés de murs oll aulres clotures équivalentes ;

$4^{\circ}$ De couper les branches d'arbres qui, se trouvant à proximité des conducteurs aériens d'électricité, pourraient, par leur mouvement ou leur chute, occasionner des courts-circuits ou des avaries aux cuvrages.

L'exécution des travanx prévus aux alinéas $1^{\circ}$ et $4^{\circ}$ ci-dessus doit être précédée d'une nolificalion directe aux intéressés et d'une enquêle spéciale dans chaque commune; elle ne peut avoir lieu qu'après approbation du projel de détail des tracés par le préfet.

Elle n'entraine aucane dépossession ; la pose d'appuis sur les murs ou facades ou sur les bils on terrasses des batiments ne peut faire obstacte au droit du propriélaire de démolir, réparer ou surélever. La pose des canalisalions ou supports dans un terrain ouvert et non bâli ne fait pas non plus obstacle au droit du propriétaire de se clore ou de batir. Le propriétaire devra, un mois avant dentreprendre les travaux de démolition, réparation, surélévation, cloture ou baliment, prévenir le concessionnaire par lettre recommandée adressée au domicile élu par ledit concessionnaire. 
Les indernnilés qui pourraient ètre dues à raison des servitudes a'appui, de passage ou d'ébranchage, prévues aux alinéas $1^{\circ}, 2^{\circ}, 3^{\circ}$ el $4^{\circ}$ ci-dessus, sonl réglées en premier ressort par le juge de paix : s'il y a expertise, le juge ne peut nommer qu'un seul expert.

Cet article reproduit, avec quelques modifications, les disposilions des articles $7,8,9$ el ro du texte de la précédente législalure.

I.es deux premiers paragraphes, dont la rédaction est empruntée au projet du Conseil d'Etat, assimilent les droits du concessionnaire à ceux que les lois el règlements donnent a l'Administration en matière de travaux publics.

Les paragr aphes suivants tomnent aux concessionnaires le droul d'appui sur les maisons, le droil de passage au-dessus des proprietés privées, et le droit d'élablissements de poteaux ou de conducteurs soulerrains sur les terrains non bâtis el non clos de murs. Ces disposilions faisaient déjà l'objel de l'arlicle 7 de la précédente législalure.

Votre Commission a cru devoir y ajouter le droit d'ébranchage, qui est conféré par la loi suisse du 24 juin rgoz el répond à un vérilable besoin, en raison des dangers que peuvent causer des courts-cireuits produit par le contact de branches mouillées avec les fils conducteurs. Larticle 4 h de cetic Ioi porte que les branches d'arbres menaçanl la sécurité ou te fonctionnement d'une ligne à faible ou à fort courant doivent, sur la demande de l'entreprise, être enlevées par le promiétaire de l'arbre movennant une indemnité.

On remarquera que le droil d'établir des supports et ancrages a élé restreint par votro Commission, qui a tenu à spécificr que l'exercice de eo droil serait limité à des courants électriques lels que la présence des conducleurs ne soil pas de nalure à présenter, malgré les précautions prises, des dangers graves pour les personnes ou les bâtiments. Elle a voulu ainsi indiquer que ce droit ne saurail être conféré aux courants à très forte tension, dont on fait un fréquent usage aujourd'hui quand il s'agit de transporter l'énergie à des distances considérables.

Ces additions ont été faites dans le seul but de sauvegarder les intérêts des propriétaires. Le texte de la proposition de loi fait peser sur la propriété quelques serviludes nouvelles Il était essentiel d'empècher que ces serviludes ne puissent devenir une cause de danger.

l.es deux paragraphes suivants de l'article 12 sc bornent à reproduire, avec des changements insignifiants, l'arlicle 8 du texte de la prícédente législature.

Le principe posé par le premier paragraphe ćlait déjà prévu par l'article 9 du lexte de la précédente législature. On a voulu rendre aussi simple que possible la procédure rolalive au règlement des indemulés pouvant êlre dues à raison des serviludes nouvelles.

\section{IITRE VI}

CONBITONS COMMUNES A L'FTABLISSEMENT ET A L'EXPIOTTHOTON

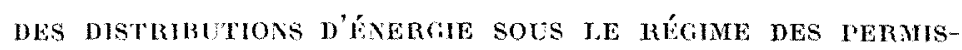
SIONS DE YOHHE OU DES CONCERSJONS.

Art. 1.3. - L'établisscment el l'exploitation des lignes de transport dénergie álectrique placécs sous le régime, soil du titre III, soil du titre IV, soit du titre $Y$ de la présente loi, sont soumises aux conditions ci-après :
Votre Commission a groupé dans le titre VI toutes les disposilions communes aux disposilions d'énergie établies sous le régime des permissions de voirie, des concessions simples et des concessions déclarées d'utilité publique.

Art. x4. - Les projets sonl examinés par les représentants des scrvices intéressés dans une conférence à laquelle prennent pout, dans tous les cas, les représentants de l'administration des postes et des télégraphes. Si l'accord en vale de l'exéculion des projets n'intervient pas au cours de la conférence, l'affaire est sóumise an comité d'électricité. Si lous les ministres inléressés n'adhèrent pas à l'avis du comité, il est slatue par décret en conseil des ministres.

Cet arlicle nouveau, inscrit à la demande des Ministres des Travaux publics et des Postes et Télégraphes, fait l'application aux distributions d'énergie électrique des règles actuellement suivies en matière de travaux publics nécessitant des conférences mixtes avec le génie militaire. Votre Commission a cru devoir rendre obligatoire l'intervention du Comité d'électricité, dont la composition sera définie à l'article 20.

Le paragraphe 2 de l'arlicle i du lexte de ta précédente législature laissait ì un règlement d'administration publique le soin de déterminer les formes de l'instruction des projets el de leur approbation par l'autorité concédante. Cette procédure est d'ailleurs conservéc dans le texte de l'a ticle 18 de la présente proposition de loi.

Art. ı5. - La mise en service d'une distribution d'énergie b́lectrique ne peut avoir lien qu'à la suite des essais faits en présence du service du contrôle et des représentants des services intéressís, ct après délivrance, par le préfet, d'une auto. risation de circulalion du courant.

Cel article nouveau a été ajouté à la demande de l'Administration des postes et télégraphes. Il ne fait, du reste, que consacrer une situation de fait existant actuellement.

Art. 16. - Le contröle de la construction el de l'exploitation est excré sous l'aulorilé du ministre des travaux publics, soit par les agents qu'il aura délégués à cet effet lorsqu'il s'agit de concessions données par l'Etat ou de permissions pour des distributions empruntant en tout ou en partie. la grande voirie, soit par les agents délégués par les municipalilés lorsqu'il s'agit de concessions données par les communes ou les syndicals de communes on de permissions pour les distribulions n'empruntant que les voies vicinales on urbaines.

Cet article nouveau est relatif à l'organisation du contrôle des distributions d'énergie

Le paragraphe a de l'arlicle in du texte de la précédenie législature laissait à un règlement d'administration publiqué le soin de déterminer l'organisation du contrôle.

La première parlie de ce rapport a déjà indiqué les motifs pour lesquels votre Commission a cru préférable de tranelier la question par la voie législative. Il lui a semblé que l'unité de contròle était indispersable ct que celui-ci devait être cxercé sous l'autorité du Ministre des Travanx publics.

\section{(A suive)}

